\title{
Isotopomer distribution computation from tandem mass spectrometric data with overlapping fragment spectra
}

\author{
Juho Rousu $^{\mathrm{a}, *}$, Ari Rantanen ${ }^{\mathrm{b}}$, Raimo A. Ketola ${ }^{\mathrm{c}}$ and Juha T. Kokkonen ${ }^{\mathrm{c}}$ \\ ${ }^{a}$ Department of Computer Science, Royal Holloway University of London, United Kingdom \\ ${ }^{\mathrm{b}}$ Department of Computer Science, University of Helsinki, Finland \\ ${ }^{\mathrm{c}}$ VTT Processes, Finland
}

\begin{abstract}
We present a method for determination of the isotopomer distributions of metabolites from the data generated by a tandem mass spectrometer. The method is an improvement over existing method as it is able to deal with overlapping fragments in the spectra. Our experiments indicate that the new method surpasses its predecessors in separating isotopomers from each other. When using the daughter ion scanning (collision induced dissociation) mode, the method was shown to be able to constrain the isotopomer distribution of different amino acids better than two existing methods. In particular, the isotopomer distributions of three amino acids, glycine, alanine and serine, can be fully uncovered with the method. However, due to the imperfect fragmentation of molecules in the tandem mass spectrometer, isotopomer distributions of larger amino acids still cannot be fully uncovered. In tests with isotope-labelled alanine, most accurate results were obtained using multiple reaction monitoring and $15 \mathrm{eV}$ collision energy. The meausured isotopomer frequecies were in the range $99-106 \%$ of the theoretical value and the deviation between repetitions was in the range $1-10 \%$.
\end{abstract}

\section{Introduction}

Metabolic flux estimation deals with uncovering the steady-state velocities of biochemical reactions in vivo. This task arises in many fields of biological research, for example, in human neurochemistry [13], plant physiology [11] and metabolic engineering of microbes [9]. In metabolic engineering, quantitative discovery of intracellular fluxes is arguably the most important question: knowledge of the fluxes is a prerequisite to optimization of the metabolic pathways towards high yield of the product metabolite of interest [19].

In recent years the use of ${ }^{13} \mathrm{C}$-labeled substrates has proven to be a promising method to quantify intracellular fluxes of the cell when there are more than one alternative pathways between two metabolites. These tracing methods are based on measuring the isotopomer distributions, that is, the different ${ }^{13} \mathrm{C}$-isotopic versions of the metabolite with their relative abundancies. By comparing the measured isotopomer distributions to the distributions expected when using each alternative pathway, one aims to deduce information about the distribution of the fluxes among the pathways $[14,16,17,19,22]$.

\footnotetext{
${ }^{*}$ Corresponding author: Juho Rousu, Department of Computer Science, Royal Holloway University of London, Egham Hill, Egham, Surrey, TW20 0EX, United Kingdom. Tel.: +44 7708235 833; E-mail: juho@cs.rhul.ac.uk.
} 
At the moment there exists two principal methods to determine the positional isotopomer distribution of the atoms, Nuclear Magnetic Resonance (NMR) and Mass Spectrometry (MS) coupled with liquid (LC-MS) or gas (GC-MS) chromatographic separation. NMR has been used for flux discovery by several authors [3,9,10,18,20,21,26]. Mass spectrometry has been applied, for example, by Di Donato et al. [4] and by Wittmann and Heinzle [24,25]. The methods also can be combined to gain more information about the positional isotopomer distribution and thus possibly about the fluxes as well $[1,12]$.

Matrix based methods for calculating positional isotopomer distributions from the data produced by MS have been developed by Wittmann and Heinzle [24], by van Winden [23] and by Dauner and Sauer [2], who all use correction matrices to remove the effect of natural labeling from measured mass spectra, and by Christensen and Nielsen [1]. These methods were brought to tandem mass spectrometers with daughter ion scanning by Rantanen et al. [15] and by Jeffrey et al. [6].

A mass isotopomer distribution obtained from mass spectrometry imposes linear constraints to the isotopomer distribution. In tandem MS, the ions can be fragmented and the fragments measured separately which increases the number of constraints. With daughter ion scanning, only one mass isotopomer is fragmented at the time that still increases the number of constraints to isotopomer distribution. In the best case with tandem MS one can deduce the isotopomer distribution in its entirety, which is not possible in the normal MS even in principle.

This paper improves the method described in [15] by allowing the isotopomers of different fragment overlap in the spectra. The structure of the article is the following. In Section 2, we shortly introduce the mass spectrometric methods used Section 3 gives a thorough description of the computational methods used to uncover the isotopomer distributions. In Section 4, experiments on discovery of isotopomer distributions of amino acids are presented. Section 5 concludes the article with discussion.

\section{Mass spectrometric methods}

Mass spectrometry is nowadays a target of interest when analysing isotopomer distributions of the metabolites because the advantage of the mass spectrometric methods compared to NMR is the sensitivity and speed. At the moment, the main mass spectrometric method used is GC-MS with electron impact (EI) ionization and a full scan mode. With this method, all the mass isotopomers of the metabolite molecule fragmentate simultaneously in the ionization chamber because of high energy, and a set of fragment ions are observed in the spectrum. When using GC-tandem mass spectrometry method (GC-MS/MS), the isotopomer ion of interest can be chosen and fragmented further (daughter ion scanning) to get additional information. Because many of the metabolites analysed are quite polar, derivatisation is needed to convert the metabolites volatile enough for GC-MS analysis. In isotopomer distribution calculations, the atoms from derivatising reagent have to also be taken account.

With an LC-MS method, when an electrospray ionisation (ESI) is used when analysing polar metabolites (in our case, amino acids), usually no derivatisation is needed which makes sample pretreatment easier and quicker. ESI is a soft technique, i.e. in a full scan mode, usually only $[\mathrm{M}+\mathrm{H}]^{+}$ion (positive ionisation mode) or $[\mathrm{M}-\mathrm{H}]^{-}$ion (negative ionisation mode) with isotopomer signals of the molecule are observed. In order to obtain fragmentation, a tandem mass spectrometer with a daughter ion scanning technique has to be used. In this technique, only one mass isotopomer is fragmented at a time which adds selectivity. However, attention has to paid to the collision energy and collision gas pressure to get optimal fragmentation conditions: the more collision energy and higher gas pressure are used, the more molecule fragments, which influences the isotopomer spectrum. This rule also applies to a GC-MS/MS technique. 
Besides the daughter ion scanning technique, also another tandem mass spectrometric method, called multiple reaction monitoring (MRM), can be used. With this method, only the daughter fragments of the molecule having the known masses are monitored while with the daughter ion scanning method the whole mass range is scanned. With a MRM-method, the sensitivity is better than with a daughter ion scanning technique which can be important when analysing isotopomer distributions of molecules at low concentrations.

In our experiments, ESI-MS- and daughter ion spectra of 20 amino acids have been measured at three different collision energies and both the structures and relative intensities of the fragments of amino acids have been determined. In addition, LC-MS and LC-MS/MS repeatability tests have been done with labelled alanine standard solutions, which consist of natural alanine, $1-{ }^{13} \mathrm{C}$-alanine and $3-{ }^{13} \mathrm{C}$-alanine.

\section{Computational method}

In the following, we derive the computational method for uncovering isotopomer distributions of molecules with a tandem mass spectrometer. We begin by a rigorous definition of the probability model of isotopomer analysis, treating fragmentation and isotopomers as probabilistic events quantified by random variables. The outline of correcting for natural abundance of non-carbon isotopes is presented next, followed by a presentation of handling the tandem mass spectra in a way that makes it possible to handle overlaps of different fragments. Equation systems for both full scan and daughter ion scanning modes are presented.

\subsection{Modeling preliminaries}

We treat a molecular species $\mathcal{M}=\{1, \ldots,|\mathcal{M}|\}$ as a sequence of numbered atom locations, each with a specific element type $E_{i} \in\{\mathrm{H}, \mathrm{He}, \mathrm{Li}, \ldots\}$. By $\mathcal{C} \subset \mathcal{M}$ and $\overline{\mathcal{C}} \subset \mathcal{M}$, we denote the sets of carbon and non-carbon locations, respectively. A fragment (species) $\mathcal{F} \subset \mathcal{M}$ is a subset of $\mathcal{M}$. By $\mathcal{C} \cap \mathcal{F}$ and $\overline{\mathcal{C}} \cap \mathcal{F}$ we denote the carbon and non-carbon locations within $\mathcal{F}$, respectively (Fig. 1). The mass/charge value of a fragment $\mathcal{F} \subseteq \mathcal{M}$ is denoted by $\mathrm{m}_{\mathrm{z}}(\mathcal{F})$.

We associate with each atom location $1 \leqslant i \leqslant|\mathcal{M}|$ a random variable $\lambda_{i}: \Omega \mapsto \mathbb{N}$ where the basic set $\Omega$ is a fixed pool of molecules of species $\mathcal{M}$. The variable $\lambda_{i}(\omega)$ denotes the isotope in location $i$ of a single randomly drawn molecule $\omega ; \lambda_{i}(\omega)=0$ denotes the lowest mass isotope of element type $E_{i}$, $\lambda_{i}(\omega)=1$, denotes the isotope with one additional neutron, and so on. In the case of carbon, we have $\lambda_{i}(\omega)=0$ for ${ }^{12} \mathrm{C}$ and $\lambda_{i}(\omega)=1$ for ${ }^{13} \mathrm{C}$.

The $\ell$ th mass isotopomer of molecular species $\mathcal{M}$ is the event ${ }^{+\ell} \mathcal{M}(\omega)=\left\{\sum_{i} \lambda_{i}(\omega)=\ell\right\}$ denoting that the atom locations within $\mathcal{M}$ contain a total of $\ell$ extra neutrons. Respectively, the $k$ th mass isotopomer of fragment $\mathcal{F}$ is the event ${ }^{+k} \mathcal{F}(\omega)=\left\{\sum_{i \in \mathcal{F}} \lambda_{i}(\omega)=k\right\}$.

(a)

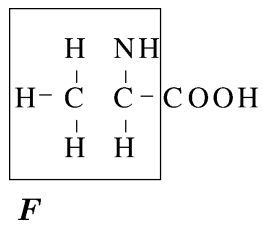

(b)

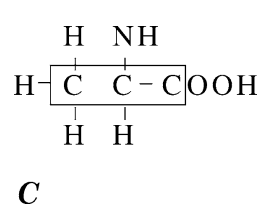

(c)

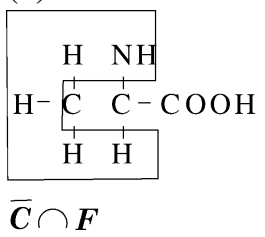

(d)

$$
\begin{array}{rl}
\mathrm{H} & \mathrm{NH} \\
\mathrm{I} & 1 \\
\mathrm{H}-\mathrm{C} & \mathrm{C}-\mathrm{COOH} \\
\mathrm{I} & 1 \\
\mathrm{H} & \mathrm{H} \\
\boldsymbol{C} \cap \overline{\boldsymbol{F}} &
\end{array}
$$

Fig. 1. Illustration of the set notation with the alanine amino acid: a fragment (a), the set of carbons (b), the set of non-carbons within the fragment (c) and the set of carbons outside the fragment (d). 
By ${ }^{+i}\{\mathcal{C} \cap \mathcal{F}\}(\omega)$ (resp. ${ }^{+i}\{\overline{\mathcal{C}} \cap \mathcal{F}\}(\omega)$ ) we denote the event that the carbons (resp. non-carbons) of $\omega$ within the fragment $\mathcal{F}$ contain $i$ extra neutrons. The $\left({ }^{13} \mathrm{C}\right)$ mass isotopomer distribution of a molecular species $\mathcal{M}$ with the carbon locations $\mathcal{C}=\left\{c_{1}, \ldots, c_{n}\right\}$, is the vector

$$
\mathbb{M}_{\mathcal{M}}=\left[\mathbb{P}\left\{{ }^{+0} \mathcal{C}\right\}, \mathbb{P}\left\{{ }^{+1} \mathcal{C}\right\}, \ldots, \mathbb{P}\left\{{ }^{+n} \mathcal{C}\right\}\right]^{T} .
$$

An $\left({ }^{13} \mathrm{C}\right)$ isotopomer is a joint event

$$
{ }^{\mathrm{w}} \mathcal{C}(\omega)=\left\{\lambda_{c_{1}}(\omega)=w_{1}\right\} \wedge\left\{\lambda_{c_{2}}(\omega)=w_{2}\right\} \wedge \cdots \wedge\left\{\lambda_{c_{n}}(\omega)=w_{n}\right\},
$$

where $\mathbf{w}=w_{1}, \ldots, w_{n}$ is a binary string since we assume that ${ }^{13} \mathrm{C}$ is the only higher carbon isotope. The vector of relative isotopomer frequencies

$$
\mathbb{I}_{\mathcal{M}}=\left[\mathbb{P}\left\{{ }^{0 \ldots 0} \mathcal{C}\right\}, \mathbb{P}\left\{{ }^{0 \ldots 1} \mathcal{C}\right\}, \ldots, \mathbb{P}\left\{{ }^{1 \ldots 0} \mathcal{C}\right\}, \mathbb{P}\left\{{ }^{1 \ldots 1} \mathcal{C}\right\}\right]^{T}
$$

is called the $\left({ }^{13} \mathrm{C}\right)$ isotopomer distribution of $\mathcal{M}$. Note that, for clarity, we usually drop the argument $\omega$ from the notation.

\subsection{Correction of natural abundance of isotopes}

Correlating the mass spectrum with the ${ }^{13} \mathrm{C}$ isotopomer distribution is hindered by the fact that other atoms types than carbon also contain - from natural causes - higher isotopes. To counteract their effect, we need to estimate for fragments $\mathcal{F} \subseteq \mathcal{M}$ the probabilites

$$
\alpha_{\mathcal{F}}(\ell)=\mathbb{P}\{+\ell\{\overline{\mathcal{C}} \cap \mathcal{F}\}\}
$$

of $\ell$ extra neutrons to recide within the non-carbons of fragment. This is accomplished by the wellknown methodology introduced by Lee et al. [8]. In the estimation, standard assumptions are made: higher isotopes of atom types other than carbon occur in a constant frequency, as reported in tables of natural abundance. Moreover, it is assumed that the isotopes occur independently from each other and, in particular, from the carbon isotopes. Based on these assumptions, (3) can be computed by considering each combination of placing $l$ extra neutrons among the non-carbons and summing the their probabilites $[8,15]$. One should note that when using derivatisation in GC-MS, the carbons of the derivating reagent need to be treated separately form the carbons of the analysed molecule $\mathcal{M}$, and, in essense, the same as the non-carbon atoms: assuming occurrence of ${ }^{13} \mathrm{C}$ isotopes with natural abundance and independently from other atoms. In practise, for large $\ell, \alpha_{\mathcal{F}}(\ell)$ will be negligible and can be taken as zero. Also note that a coefficient $\alpha_{\mathcal{F}}(\ell)$ can only be non-zero for $0 \leqslant \ell \leqslant N_{\mathcal{F}}$, where $N_{\mathcal{F}}$ is the maximum number of extra neutrons $\mathcal{F}$ can accommodate.

Handling a mass spectrum containing only peaks corresponding to an unfragmented molecular species $\mathcal{M}$ is straightforward given the precomputed coefficients ${ }^{+\ell_{\alpha}} \alpha_{\mathcal{F}}$. Denote with $m$ the base mass of the molecular species $\mathcal{M}$, with $h(t)$ the intensity of a peak with $\mathrm{m}_{\mathrm{z}}=t$ and with $N$ the maximum number of extra neutrons that the molecular species can accommodate. The relative intensity $\iota$ can then be interpreted as the relative frequency of the corresponding mass isotopomer:

$$
\iota(m+\ell)=\frac{h(m+\ell)}{\sum_{t=m}^{m+N} h(t)} \simeq \mathbb{P}\left\{{ }^{+\ell} \mathcal{M}\right\} .
$$


Assuming that the carbon and non-carbon isotopes occur independently from another, we can decompose the above into a weighted sum of carbon mass isotopomer probabilites

$$
\mathbb{P}\left\{{ }^{+\ell} \mathcal{M}\right\}=\sum_{i=0}^{\ell} \mathbb{P}\left\{{ }^{+\ell-i} \overline{\mathcal{C}}\right\} \cdot \mathbb{P}\left\{{ }^{+i} \mathcal{C}\right\}=\sum_{i=0}^{\ell} \alpha_{\mathcal{M}}(\ell-i) \cdot \mathbb{P}\left\{{ }^{+i} \mathcal{C}\right\}
$$

The equation sums over the probabilities of all possible combinations of putting $i$ extra neutrons among the carbons and the rest $\ell-i$ extra neutrons among the non-carbons.

Each carbon mass isotopomer ${ }^{+i} \mathcal{C}$ can be further decomposed into a sum of (positional) isotopomers

$$
\mathbb{P}\left\{{ }^{+i} \mathcal{C}\right\}=\sum_{\left\{\mathbf{w} \mid \sum_{p} w_{p}=i\right\}} \mathbb{P}\left\{{ }^{\mathbf{w}} \mathcal{C}\right\}
$$

Plugging the above to (4) and noticing that each isotopomer ${ }^{\mathrm{w}} C$ appears exactly once in the sum gives us the desired linear equation between the carbon isotopomers probabilites and the measured relative peak intensity:

$$
\iota(m+\ell)=\sum_{i=0}^{\ell} \sum_{\left\{\mathbf{w} \mid \sum_{p} w_{p}=i\right\}} \alpha_{\mathcal{M}}(\ell-i) \cdot \mathbb{P}\left\{{ }^{\mathbf{w}} C\right\}=\sum_{\mathbf{w} \in\{0,1\}^{n}} \alpha_{\mathcal{M}}\left(\ell-\sum_{p} w_{p}\right) \mathbb{P}\left\{{ }^{\mathbf{w}} C\right\} .
$$

The same procedure is repeated for each peak $t \in\{m, \ldots, m+N\}$ to obtain a system of linear equations

$$
A \cdot \mathbb{I}_{\mathcal{M}}=\left[\begin{array}{ccc}
\alpha(0,0) & \ldots & \alpha\left(0,2^{n}-1\right) \\
\vdots & \ddots & \vdots \\
\alpha(N, 0) & \ldots & \alpha\left(N, 2^{n}-1\right)
\end{array}\right] \cdot\left[\begin{array}{c}
\mathbb{P}\left\{{ }^{0 \ldots 0} \mathcal{C}\right\} \\
\vdots \\
\mathbb{P}\{1 \ldots 1 \\
\left.{ }^{1 \ldots 1}\right\}
\end{array}\right]=\left[\begin{array}{c}
\iota(m) \\
\vdots \\
\iota(m+N)
\end{array}\right]=\vec{\iota}_{\mathcal{M}}
$$

where we denoted $\alpha(\ell, q)=\alpha_{\mathcal{M}}\left(\ell-\sum_{p} w_{p}\right)$ where $\mathbf{w}$ is the $q$ th binary string of length $n$. The matrix $A$ is less than full rank for any molecule that contains more than one carbon. This is because two molecules of the same weight fall into the same peak, regardless of the location of the labels. Thus, from (5) we can solve the mass isotopomer distribution (1) but not the isotopomer distribution (2). Fortunately, tandem mass spectrometry can help us in obtaining better solutions.

\subsection{Utilizing tandem mass spectrometry}

The full scan-mode tandem mass spectrum contains all produced fragments of all ions, regardless of their mass. The daughter ion scanning-mode spectra are more selective, only containing fragments originating from ions of a particular mass. Both methods enable us to obtain further information of the isotopomers that have the same number of labels.

Methods for the full scan mode have been previously presented by Christensen and Nielsen [1] and methods for daughter ion scanning by Rantanen et al. [15] and Jeffrey et al. [6]. In this paper we extend the previous works by considering the problem of overlaps in the daughter spectra in a systematic manner.

Overlaps in the fragment spectra complicate the isotopomer analysis. An overlap occurs when two or more fragments have their mass close together, for example, the fragments $\mathrm{C}_{2} \mathrm{H}_{3}(\mathrm{~m} / \mathrm{z}=27)$ and $\mathrm{CH}_{3} \mathrm{~N}$ 
$(\mathrm{m} / \mathrm{z}=29)$ overlap since the mass isotopomer ${ }^{+\ell+2}\left\{\mathrm{C}_{2} \mathrm{H}_{3}\right\}$ has the same (integral) mass as the mass isotopomer ${ }^{+}\left\{\mathrm{CH}_{3} \mathrm{~N}\right\}$, where $\ell \in\{1,2, \ldots\}$. The contribution of one fragment to the peak depends, in addition to the isotopomer distribution of the molecule, on the probability that the fragment emerged in the mass spectrometer.

For GC-MS, consideration of overlaps has been performed by Jeffrey et al. [6]. They, however, present only the case where the overlapping fragments are known to originate from different source molecules. This may be warranted assuming that the fragments of a derivatized molecule have distinctly different weights.

In the following, we derive a method for tackling the overlaps in a general manner, without the above assumption. For each fragment $\mathcal{F} \subseteq \mathcal{M}$, let us define a random variable $g_{\mathcal{F}}: \Omega \mapsto\{0,1\}$ and an event $G_{\mathcal{F}}=\left\{g_{\mathcal{F}}(\omega)=1\right\}$ to denote whether fragment $\mathcal{F}$ was generated out of the molecule $\omega$ or not. Consider now a tandem mass spectrum $\left(h^{0}(0), \ldots, h^{0}\left(\mathrm{~m}_{\mathrm{z}}(\mathcal{M})+N\right)\right)$ generated by sampling a pool of unlabelled molecules.

Assuming that no fragments have exactly the same mass, the expected relative intensity $\mathbb{E}\left(\iota^{0}\left(\mathrm{~m}_{\mathrm{z}}(\mathcal{F})\right)\right)=$ $\mathbb{P}\left\{G_{\mathcal{F}}\right\} / \sum_{\mathcal{F}_{i} \subseteq \mathcal{M}} \mathbb{P}\left\{G_{\mathcal{F}_{i}}\right\}$ is directly related to the emergence probability of the fragment. The observed intensity $\iota\left(\mathrm{m}_{\mathrm{z}}(\mathcal{F})\right)$ can thus be used to approximate the relative probability

$$
r_{\mathcal{F}}=\frac{\mathbb{P}\left\{G_{\mathcal{F}}\right\}}{\sum_{\mathcal{F}_{i} \subseteq \mathcal{M}} \mathbb{P}\left\{G_{\mathcal{F}_{i}}\right\}} \simeq \iota^{0}\left(\mathrm{~m}_{\mathrm{z}}(\mathcal{F})\right)
$$

of fragment $\mathcal{F}$ emerging.

\subsubsection{Equation system for the full scan mode}

Let us now examine how the situation is compicated by the isotope labeling. With LC-MS in full scan mode, usually only $[\mathrm{M}+\mathrm{H}]^{+}$ion (positive ionisation mode) or $[\mathrm{M}-\mathrm{H}]^{-}$ion (negative ion mode) with isotopomer signals of the molecule is observed. With GC-MS, in the full scan-mode, each peak generally contains isotopomers of all fragments that emerged in the ion source and have mass isotopomers of the same $\mathrm{m}_{\mathrm{z}}$ value, and correspondingly, each fragment contributes to a number of peaks.

For a peak $\mathrm{m}_{\mathrm{z}}=t$ in the spectrum, denote by $\delta_{\mathcal{F}}(t)=t-\mathrm{m}_{\mathrm{z}}(\mathcal{F})$ the offset from the fragment peak $\mathrm{m}_{\mathrm{z}}(\mathcal{F})$ and by $\mathcal{S}(t)=\left\{\mathcal{F} \subseteq \mathcal{M} \mid 0 \leqslant \delta_{\mathcal{F}}(t) \leqslant N_{\mathcal{F}}\right\}$ the set of fragments that have mass isotopomers able to contribute to the peak $\mathrm{m}_{\mathrm{z}}=t$, where $N_{\mathcal{F}}$ is the maximum number of extra neutrons the fragment can accommodate.

The contribution of fragment $\mathcal{F}$ to the expected intensity of the peak $t=\mathrm{m}_{\mathrm{z}}(\mathcal{F})+k$ is $\mathbb{P}\left\{{ }^{+k} \mathcal{F} \cap G_{\mathcal{F}}\right\}=$ $\mathbb{P}\left\{{ }^{+k} \mathcal{F}\right\} \cdot \mathbb{P}\left\{G_{\mathcal{F}}\right\}$ assuming negligible isotope effects, that is, the emergence of certain fragment is independent of which isotopomer is in question. The probability of a fragment of mass/charge $\mathrm{m}_{\mathrm{z}}=t$ emerging can then be expressed as

$$
\sum_{\mathcal{F} \in \mathcal{S}(t)} \mathbb{P}\left\{{ }^{+\delta_{\mathcal{F}}(t)} \mathcal{F} \cap G_{\mathcal{F}}\right\}=\sum_{\mathcal{F} \in \mathcal{S}(t)} \mathbb{P}\left\{{ }^{+\delta_{\mathcal{F}}(t)} \mathcal{F}\right\} \mathbb{P}\left\{G_{\mathcal{F}}\right\}
$$

and the peak's expected proportion of the total intensity of the spectrum as

$$
\sum_{\mathcal{F} \in \mathcal{S}(t)} \mathbb{P}\left\{{ }^{+\delta_{\mathcal{F}}(t)} \mathcal{F}\right\} \frac{\mathbb{P}\left\{G_{\mathcal{F}}\right\}}{\sum_{\mathcal{F}_{i} \subseteq \mathcal{M}} \mathbb{P}\left\{G_{\mathcal{F}_{i}}\right\}}=\sum_{\mathcal{F} \in \mathcal{S}(t)} \mathbb{P}\left\{{ }^{+\delta_{\mathcal{F}}(t)} \mathcal{F}\right\} \cdot r_{\mathcal{F}},
$$


assuming that isotope labeling does not affect the fragment emergence probabilities $r_{\mathcal{F}}$. The factor $\mathbb{P}\left\{{ }^{+\delta_{\mathcal{F}}(t)} \mathcal{F}\right\}$ can be decomposed by considering all possible ways of allocating the $k=\delta_{\mathcal{F}}(t)$ extra neutrons among the carbons and non-carbons of the fragment:

$$
\mathbb{P}\left\{{ }^{+k} \mathcal{F}\right\}=\sum_{i=0}^{k} \mathbb{P}\left\{{ }^{+k-i}\{\overline{\mathcal{C}} \cap \mathcal{F}\}\right\} \cdot \mathbb{P}\left\{{ }^{+i}\{\mathcal{C} \cap \mathcal{F}\}\right\}=\sum_{i=0}^{k} \alpha_{\mathcal{F}}(k-i) \cdot \mathbb{P}\left\{{ }^{+i}\{C \cap F\}\right\}
$$

Furthermore, we can decompose $\mathbb{P}\left\{{ }^{+i}\{\mathcal{C} \cap \mathcal{F}\}\right\}$ as a sum of (positional) carbon isotopomers of the molecule $\mathcal{M}$ that have $i=i_{\mathcal{F}}(\mathbf{w})=\sum_{p \in \mathcal{C} \cap \mathcal{F}} w_{p}$ labels among the carbons within the fragment:

$$
\sum_{i=0}^{k} \alpha_{\mathcal{F}}(k-i) \cdot \mathbb{P}\left\{{ }^{+i}\{\mathcal{C} \cap \mathcal{F}\}\right\}=\sum_{\mathbf{w} \in\{0,1\}^{n}} \alpha_{\mathcal{F}}\left(k-i_{\mathcal{F}}(\mathbf{w})\right) \cdot \mathbb{P}\left\{{ }^{\mathbf{w}} \mathcal{C}\right\} .
$$

We equate the expected and the observed relative intensities to obtain an equation for a peak $\mathrm{m}_{\mathrm{z}}=t$ in the full scan mode spectrum. We thus obtain

$$
\begin{aligned}
\iota_{F S}(t) & =\sum_{\mathcal{F} \in \mathcal{S}(t)} \sum_{\mathbf{w} \in\{0,1\}^{n}} \alpha_{\mathcal{F}}\left(\delta_{\mathcal{F}}(t)-i_{\mathcal{F}}(\mathbf{w})\right) \cdot \mathbb{P}\left\{{ }^{\mathbf{w}} \mathcal{C}\right\} \cdot r_{\mathcal{F}} \\
& =\sum_{\mathbf{w} \in\{0,1\}^{n}} \mathbb{P}\left\{{ }^{\mathbf{w}} \mathcal{C}\right\} \sum_{\mathcal{F} \in \mathcal{S}(t)} \alpha_{\mathcal{F}}\left(\delta_{\mathcal{F}}(t)-i_{\mathcal{F}}(\mathbf{w})\right) \cdot r_{\mathcal{F}}=\sum_{\mathbf{w} \in\{0,1\}^{n}} \mathbb{P}\left\{{ }^{\mathbf{w}} \mathcal{C}\right\} \cdot \beta(t, \mathbf{w}),
\end{aligned}
$$

by changing the order of summation, taking $\mathbb{P}\left\{{ }^{\mathbf{w}} \mathcal{C}\right\}$ out of the inner sum and finally denoting $\beta(t, \mathbf{w})=$ $\sum_{\mathcal{F} \in \mathcal{S}(t)} \alpha_{\mathcal{F}}\left(\delta_{\mathcal{F}}(t)-i_{\mathcal{F}}(\mathbf{w})\right) \cdot r_{\mathcal{F}}$.

The linear equations thus obtained are collected into a matrix system

$$
B_{F S} \cdot \mathbb{I}_{\mathcal{M}}=\left[\begin{array}{ccc}
\beta(0,0) & \cdots & \beta\left(0,2^{n}-1\right) \\
\vdots & \ddots & \vdots \\
\beta(m+N, 0) & \cdots & \beta\left(m+N, 2^{n}-1\right)
\end{array}\right] \cdot \mathbb{I}_{\mathcal{M}}=\left[\begin{array}{c}
\iota_{F S}(0) \\
\vdots \\
\iota_{F S}(m+N)
\end{array}\right]=\vec{\iota}_{F S}
$$

denoting $\beta(t, q)=\beta(t, \mathbf{w})$ where $\mathbf{w}$ is the $q$ th binary string of length $n$.

\subsubsection{Equation system for the daughter ion scanning-mode}

In daughter ion scanning, one selects a single mass isotopomer ${ }^{+} \ell \mathcal{M}$ to be subsequently fragmented. Thus the daughter ion spectrum contains fragments that originated from a molecules of equal weight. This procedure is then repeated for all interesting mass isotopomers of the molecule, which results in a collection of daughter spectra.

Selecting the mass isotopomer ${ }^{+} \ell \mathcal{M}$ implies that the daughter peak $\mathrm{m}_{\mathrm{z}}=t$ contains those fragment mass isotopomers ${ }^{+k} \mathcal{F}$ only that have correct weight, that is $\mathrm{m}_{\mathrm{z}}(\mathcal{F})+k=t$ and that originate from the selected molecule ion. The relative intensity of the daughter peak satisfies

$$
\iota_{D}(t, \ell)=\sum_{\mathcal{F} \in \mathcal{S}(t)} \mathbb{P}\left\{\left.{ }^{+k} \mathcal{F}\right|^{+\ell} \mathcal{M}\right\} \cdot r_{\mathcal{F}}
$$

Note that the probability of the fragment $\mathcal{F}$ emerging is taken to be independent of which parent ion was selected. The factor $\mathbb{P}\left\{{ }^{+k} \mathcal{F} \mid{ }^{+\ell} \mathcal{M}\right\}=\mathbb{P}\left\{{ }^{+k} \mathcal{F} \cap{ }^{+\ell} \mathcal{M}\right\} / \mathbb{P}\left\{{ }^{+\ell} \mathcal{M}\right\}$ can be further decomposed into a 
sum of probabilities of events where $i$ labels are situated within the carbons of the fragment, $k-i$ labels within the non-carbons of the fragment, $j$ labels among the carbons outside the fragment and $l-k-j$ labels among the rest of the atoms:

$$
\begin{aligned}
\mathbb{P}\left\{\left.{ }^{+k} \mathcal{F}\right|^{+\ell} \mathcal{M}\right\} & =\frac{\sum_{i=0}^{k} \sum_{j=0}^{\ell-k} \mathbb{P}\left\{{ }^{+i}\{\mathcal{C} \cap \mathcal{F}\} \cap{ }^{+j}\{\mathcal{C} \cap \overline{\mathcal{F}}\} \cap{ }^{+k-i}\{\overline{\mathcal{C}} \cap \mathcal{F}\} \cap{ }^{+\ell-k-j}\{\overline{\mathcal{C}} \cap \overline{\mathcal{F}}\}\right\}}{\mathbb{P}\left\{{ }^{+\ell} \mathcal{M}\right\}} \\
& =\sum_{i=0}^{k} \sum_{j=0}^{\ell-k} \mathbb{P}\left\{{ }^{+i}\{\mathcal{C} \cap \mathcal{F}\} \cap^{+j}\{\mathcal{C} \cap \overline{\mathcal{F}}\}\right\} \cdot \alpha_{\mathcal{F}}(\ell, k-i, \ell-k-j) .
\end{aligned}
$$

The last equality was obtained by making the same assumption are before that the labels in the non-carbon part occur independently of each other and of the labels among the carbons, and denoting $\alpha_{\mathcal{F}}(\ell, t, u)=\mathbb{P}\left\{{ }^{+t}\{\overline{\mathcal{C}}\} \cap \mathcal{F}\right\} \cdot \mathbb{P}\left\{{ }^{+u}\{\overline{\mathcal{C}} \cap \overline{\mathcal{F}}\}\right\} / \mathbb{P}\left\{{ }^{+\ell} \mathcal{M}\right\}$. Note that the numerator is given by $\mathbb{P}\left\{{ }^{+t}\{\overline{\mathcal{C}}\} \cap \mathcal{F}\right\} \cdot \mathbb{P}\left\{{ }^{+u}\{\overline{\mathcal{C}} \cap \overline{\mathcal{F}}\}\right\}=\alpha_{\mathcal{F}}(t) \cdot \alpha_{\overline{\mathcal{F}}}(u)$ and that $\mathbb{P}\left\{{ }^{+\ell} \mathcal{M}\right\} \simeq \iota\left(\mathrm{m}_{\mathrm{z}}(\mathcal{M})+\ell\right)$ can be obtained from the spectrum of the unfragmented molecule.

As in the full scan mode, by taking advantage of the fact that each isotopomer occurs exactly once in the (double) sum we can decompose the carbon mass isotopomer probability as a sum of isotopomer probabilities

$$
\begin{aligned}
& \sum_{i=0}^{k} \sum_{j=0}^{\ell-k} \mathbb{P}\left\{{ }^{+i}\{\mathcal{C} \cap \mathcal{F}\} \cap{ }^{+j}\{\mathcal{C} \cap \overline{\mathcal{F}}\}\right\} \alpha_{\mathcal{F}}(\ell, k-i, \ell-k-j) \\
& \quad=\sum_{\mathbf{w} \in\{0,1\}^{n}} \mathbb{P}\left\{{ }^{\mathbf{w}} \mathcal{C}\right\} \alpha_{\mathcal{F}}\left(\ell, k-i_{\mathcal{F}}(\mathbf{w}), \ell-k-j_{\mathcal{F}}(\mathbf{w})\right),
\end{aligned}
$$

denoting $j_{\mathcal{F}}(\mathbf{w})=\sum_{p \in \mathcal{C} \cap \overline{\mathcal{F}}} w_{p}$ and $i_{\mathcal{F}}(\mathbf{w})=\sum_{p \in \mathcal{C} \cap \mathcal{F}} w_{p}$.

By similar derivation as in (7), the equation for the daughter peak can thus becomes

$$
\begin{aligned}
\iota_{D}(t, \ell) & =\sum_{\mathcal{F} \in \mathcal{S}(t)} \sum_{\mathbf{w} \in\{0,1\}^{n}} \mathbb{P}\left\{{ }^{\mathbf{w}} \mathcal{C}\right\} \alpha_{\mathcal{F}}\left(\ell, \delta_{\mathcal{F}}(t)-i_{\mathcal{F}}(\mathbf{w}), \ell-\delta_{\mathcal{F}}(t)-j_{\mathcal{F}}(\mathbf{w})\right) \cdot r_{\mathcal{F}} \\
& =\sum_{\mathbf{w} \in\{0,1\}^{n}} \mathbb{P}\left\{{ }^{\mathbf{w}} \mathcal{C}\right\} \cdot \beta_{\mathcal{F}}(\ell, t, \mathbf{w})
\end{aligned}
$$

where we denoted

$$
\beta_{\mathcal{F}}(\ell, t, \mathbf{w})=\sum_{\mathcal{F} \in \mathcal{S}(t)} \alpha_{\mathcal{F}}\left(\ell, \delta_{\mathcal{F}}(t)-i_{\mathcal{F}}(\mathbf{w}), \ell-\delta_{\mathcal{F}}(t)-j_{\mathcal{F}}(\mathbf{w})\right) \cdot r_{\mathcal{F}}
$$

The above equations can be collected into a system of linear equations. From the daughter ion spectrum of ${ }^{+\ell} \mathcal{M}$, we get the following system

$$
B_{\ell} \cdot \mathbb{I}_{\mathcal{M}}=\left[\begin{array}{ccc}
\beta(\ell, 0,0) & \cdots & \beta\left(\ell, 0,2^{n}-1\right) \\
\vdots & \ddots & \vdots \\
\beta(\ell, m+N, 0) & \cdots & \beta\left(\ell, m+N, 2^{n}-1\right)
\end{array}\right] \cdot \mathbb{I}_{\mathcal{M}}=\left[\begin{array}{c}
\iota_{D}(0, \ell) \\
\vdots \\
\iota_{D}(m+N, \ell)
\end{array}\right]=\vec{\iota}_{D I S}(\ell),
$$

where again $\beta(\ell, t, q)=\beta(\ell, t, \mathbf{w})$ for $q$ th length- $n$ binary string $\mathbf{w}$. 
The systems of equations for the unfragmented molecule, the full scan-mode daughter spectrum and the set of daughter spectra emerging from the daughter ion scanning experiment all represent linear constraints to the isotopomer distribution $\mathbb{I}_{\mathcal{M}}$ of the molecule. Thus they, or any subset of them, can be collected into a common system

$$
S \cdot \mathbb{I}_{\mathcal{M}}=\left[\begin{array}{c}
A \\
B_{F S} \\
B_{0} \\
\vdots \\
B_{N}
\end{array}\right] \cdot \mathbb{I}_{\mathcal{M}}=\left[\begin{array}{c}
\vec{\iota}_{M} \\
\vec{\iota}_{F S} \\
\vec{\iota}_{D I S}(0) \\
\vdots \\
\vec{\iota}_{D I S}(N)
\end{array}\right]
$$

and solved together.

\section{Experiments}

\subsection{Materials and methods}

\subsubsection{Materials}

Natural L-amino acids were obtained from Sigma-Aldrich (Steinheim, Germany). $1-{ }^{13} \mathrm{C}$ and $3-{ }^{13} \mathrm{C}$ labelled L-alanine $\left(99\right.$ atom\% $\left.{ }^{13} \mathrm{C}\right)$ were from Aldrich Chem. Co (Milwaukee WI, USA). Acetonitrile (ACN) used was from Rathburn (Walkerburn, Scotland, UK), formic acid from J.T. Baker (Deventer, The Netherlands) and heptafluorobutyric acid (HFBA) from Sigma-Aldrich (Steinheim, Germany). All the reagents and solvent were of analytical purity.

\subsubsection{L-amino acid standards}

In direct infusion experiments with microsyringe pump (no LC-separation), the amino acid solution concentrations used in mass spectra analyses were $10 \mu \mathrm{g} / \mathrm{ml}$ and they were done in acetonitrile/water $1: 1+0.1 \%$ formic acid. In addition, an alanine solution $(10 \mu \mathrm{g} / \mathrm{ml})$ where $5 \%$ was $1{ }^{13} \mathrm{C}$-alanine, $5 \%$ $3-{ }^{13} \mathrm{C}$-alanine and $90 \%$ natural alanine, was analysed.

In LC-MS experiments the amino acid solution concentrations were about $100 \mu \mathrm{g} / \mathrm{ml} \mathrm{or} 1 \mathrm{mg} / \mathrm{ml}$ in water. In addition, an alanine solution (ca. $100 \mu \mathrm{g} / \mathrm{ml}$ ) where $6.6 \%$ was $1{ }^{13} \mathrm{C}$-alanine, $6.9 \%$ $3-{ }^{13} \mathrm{C}$-alanine and $86.5 \%$ natural alanine, was analysed.

\subsubsection{Instruments}

The mass spectrometer used was Micromass Quattro II triple quadrupole instrument equipped with an electrospray interface (Manchester, UK). The spectra were measured in a positive ionisation mode. The electrospray capillary voltage was $3.5 \mathrm{kV}$ and cone voltage varied between 20-60 V. Collision induced dissociation (CID) daughter ion spectra of the amino acids were measured with three different collision energies $(15,30$ and $50 \mathrm{eV})$. Collision gas was argon with a gas pressure of $1.5 \mathrm{e}-3 \mathrm{mbar}$.

The liquid chromatograph used was HP 1100 (USA) with an autosampler. The column was XTerra MS C18, $1 \times 150 \mathrm{~mm}, 3.5 \mu \mathrm{m}$ particle size (Waters, Milford MA, USA). Eluents used were: $\mathrm{A}=0.3 \%$ HFBA $(V / V), B=A C N+0.3 \%$ HFBA $(V / V)$. A LC-gradient program was: $0 \%, 7 \mathrm{~min} \rightarrow 2.5 \% / \mathrm{min}$ $\mathrm{B} \rightarrow 50 \% \mathrm{~B}$, flow rate $40 \mu \mathrm{l} / \mathrm{min}$, injection volume $2 \mu \mathrm{l}$. 


\subsubsection{Repeatability tests}

In LC-MS and LC-MS/MS repeatability tests, an alanine solution (ca. $100 \mu \mathrm{g} / \mathrm{ml}$ ) where $6.6 \%$ was $1-{ }^{13} \mathrm{C}$-alanine, $6.9 \% 3-{ }^{13} \mathrm{C}$-alanine and $86.5 \%$ natural alanine, was used. In measurements, daughter ion scanning mode (collision energy $15 \mathrm{eV}$ ) and MRM mode with two collision energies ( $15 \mathrm{eV}$ and $30 \mathrm{eV}$ ) were tested. With the daughter ion scanning mode, collision induced dissosiation (CID) was made for alanine isotopomer signals m/z $90(\mathrm{M}+\mathrm{H}), \mathrm{m} / \mathrm{z} 91(\mathrm{MH}+1), 92(\mathrm{MH}+2)$ and $93(\mathrm{MH}+3)$. With the MRM mode, monitored fragment signals were $\mathrm{m} / \mathrm{z} 91 \rightarrow \mathrm{m} / \mathrm{z} 27-30,44,45, \mathrm{~m} / \mathrm{z} 92 \rightarrow \mathrm{m} / \mathrm{z}$ $28-30,45,46$ and $\mathrm{m} / \mathrm{z} 93 \rightarrow \mathrm{m} / \mathrm{z}$ 29, 30, 46. Calculations have been done with PIDCv2. Repeatability (RSDs, $n=5$ injections) and measured isotopomer distribution/theoretical distribution relationship were compared between these methods.

\subsection{Results of repeatability tests}

LC-MS/MS spectra of the mixture of $1-{ }^{13} \mathrm{C} / 3-{ }^{13} \mathrm{C}$-labelled alanine and natural alanine measured from different isotopomer signals are presented in Fig. 2 where the differences in the daughter ion spectra can be seen as a result of a amount of ${ }^{13} \mathrm{C}$-labelled carbons in the fragments. In the daughter ion spectrum, where $\mathrm{m} / \mathrm{z} 90$ is fragmented, there is no ${ }^{13} \mathrm{C}$-label in the molecule and the spectra where the fragmentations were done at $\mathrm{m} / \mathrm{z} 91$ and 92 , the labelled carbons are included.

The suitability of the described approach for isotopomer distribution calculations have been tested in the repeatability tests, where two LC-tandem mass spectrometric methods, daughter ion scanning and MRM method, were used for analysing the isotopomer distributions for ${ }^{13} \mathrm{C}$-labelled alanine standard mixture. With the MRM method, also the influence of collision energy was investigated and two different collision energies (15 and $30 \mathrm{eV}$ ) were used. Comparison of the measured isotopomer distribution/theoretical distribution relationship (\%) with the tandem mass spectrometric methods used, is presented in Fig. 3. It can be observed that depending on the isotopomer and the method, the measured

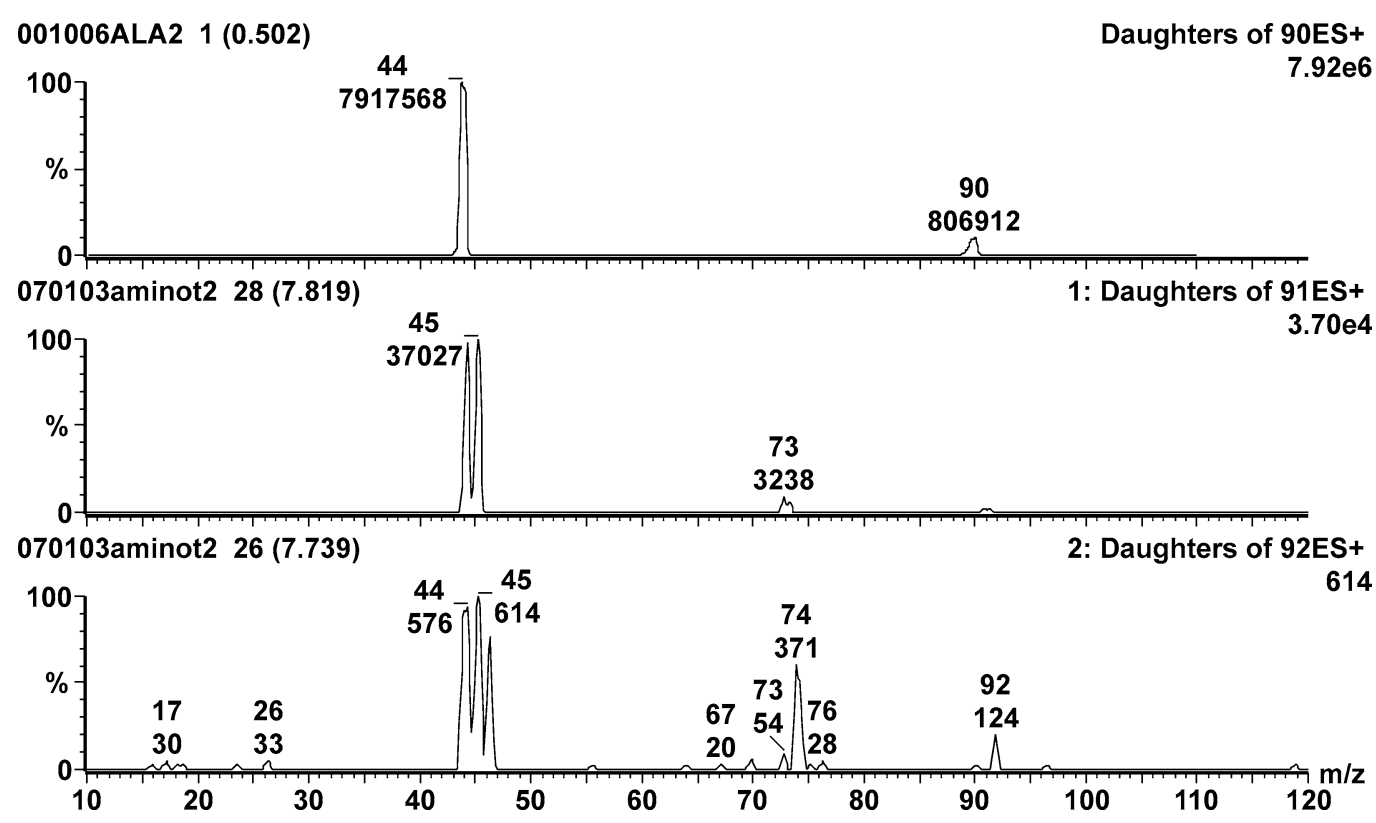

Fig. 2. LC-MS/MS spectra the mixture of $1-{ }^{13} \mathrm{C} / 3-{ }^{13} \mathrm{C}$-labelled alanine + natural alanine measured from different isotopomer signals (CID at $\mathrm{m} / \mathrm{z}$ 90-92, collision energy $15 \mathrm{eV}$ ). 
distributions varied between $83-142 \%$ from the theoretical ones. The best results were obtained with MRM method at collision energy $15 \mathrm{eV}$, the variation being between $99-106 \%$. With the daughter ion scanning mode at $15 \mathrm{eV}$, the variation was between $98-111 \%$ and with the MRM mode at $30 \mathrm{eV}$, the variation was $83-142 \%$ from the theoretical distribution. One reason for higher error with $30 \mathrm{eV}$ collision energy can be the higher noise in the spectrum, especially when fragmentating isotopomer signals of lower intensities.

The same tendency can also be observed when comparing repeatability between the methods (Fig. 4). Relative standard deviations (RSD) for the calculated isotopomer distributions between the injections of

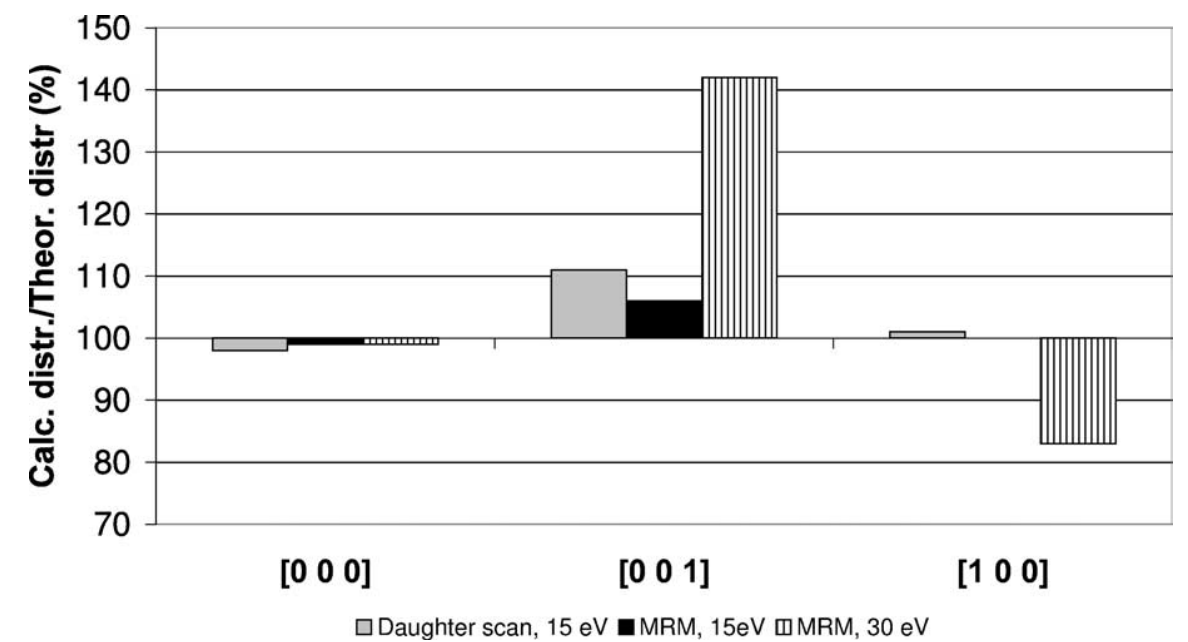

Fig. 3. Comparison of different mass spectrometric methods used for a determination of positional isotopomer distributions (calculated by PIDCv2) from the ${ }^{13} \mathrm{C}$-labelled alanine solutions, in which the calculated distribution are compared to the theoretical distributions. $\left[\begin{array}{lll}0 & 0 & 0\end{array}\right]=$ unlabelled alanine, $\left[\begin{array}{lll}0 & 0 & 1\end{array}\right]={ }^{13} \mathrm{C}$-label in carbon number $3,\left[\begin{array}{lll}1 & 0 & 0\end{array}\right]={ }^{13} \mathrm{C}-$ label in carbon number 1 . Carbon numbering of amino acids follows IUPAC guidelines.

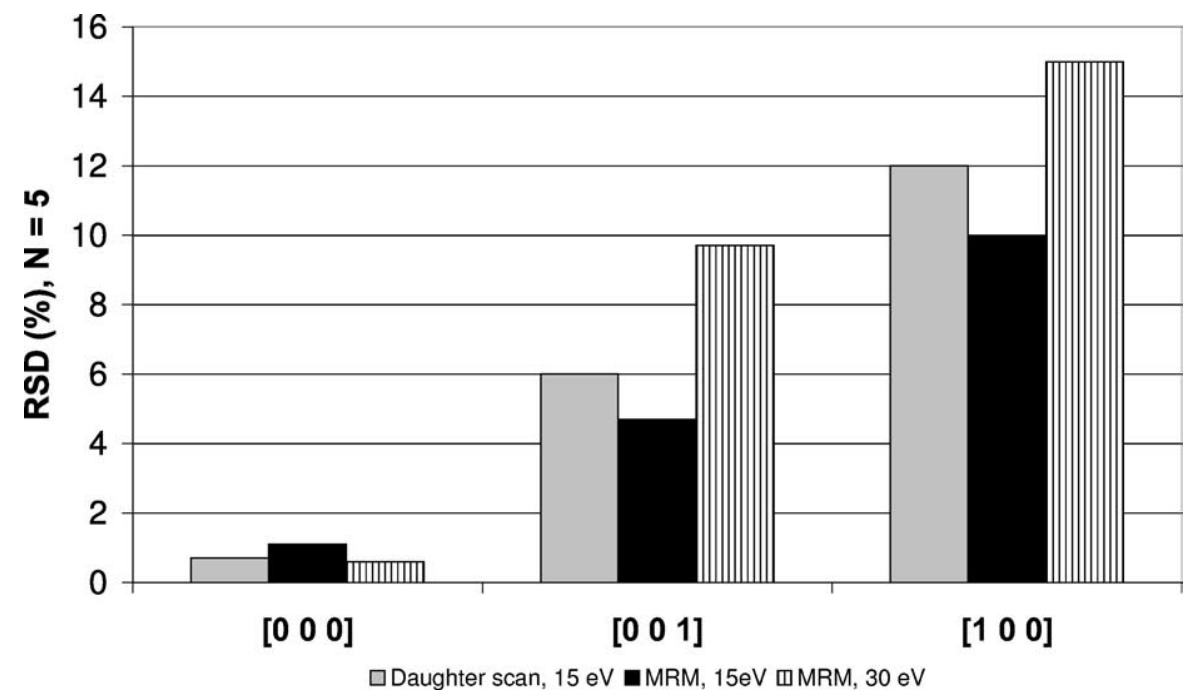

Fig. 4. Comparison of repeatability (RSD) of different mass spectrometric methods used for a determination of positional isotopomer distributions (calculated by PIDCv2) from the ${ }^{13} \mathrm{C}$-labelled alanine solutions ( $N=5$ injections/method). 
labelled alanine solution, is the best with the MRM mode at $15 \mathrm{eV}$, the RSDs varying between 1.1-10\% depending on the isotopomer. For the daughter ion scanning mode at $15 \mathrm{eV}$, the RSDs were between $0.7-12 \%$ and for MRM mode at $30 \mathrm{eV}$, the RSDs were between $0.6-15 \%$.

\subsection{Identifiability of isotopomer distributions}

The extent to which an isotopomer distribution is identifiable from tandem mass spectrometric data depends on two aspects: First, the frequencies of mass isotopomers need to be sufficient the corresponding peaks to be reliably detected. Second, the fragmentation of the molecule needs to be sufficient. In order to completely pinpoint the abundance of each isotopomer, for every pair of carbons there needs to exists a fragment where exactly one of the carbons appears. For a set of carbons $\mathcal{C}^{\prime}$ that always occur together it is not possible to distinguish between the isotopomers of the molecule $\mathcal{M}$ where the only difference is the location of the labels within $\mathcal{C}^{\prime}$. In the best case, we can give an equation giving the combined abundance of such a group. The number of such groups in the output is given by the rank of the equation system constructed from the measurements. In the case of a completely determined isotopomer distribution each group contains a single (different) isotopomer.

In Fig. 5 we compare three methods in the analysis of isotopomer distributions of amino acids: the LC-MS daughter-ion scanning methods presented in this paper (leftmost column) and that of Rantanen et al. [15] (middle column), and the results by Dauner and Sauer [2] on a GC-MS-based method (rightmost column). The vertical axis denotes the relative rank of the equation system compared to the theoretical maximum $2^{|\mathcal{C}|}$ corresponding to completely determined isotopomer distribution.

LC-MS/DIS+Overlap handling DLC-MS/DIS EGC-MS

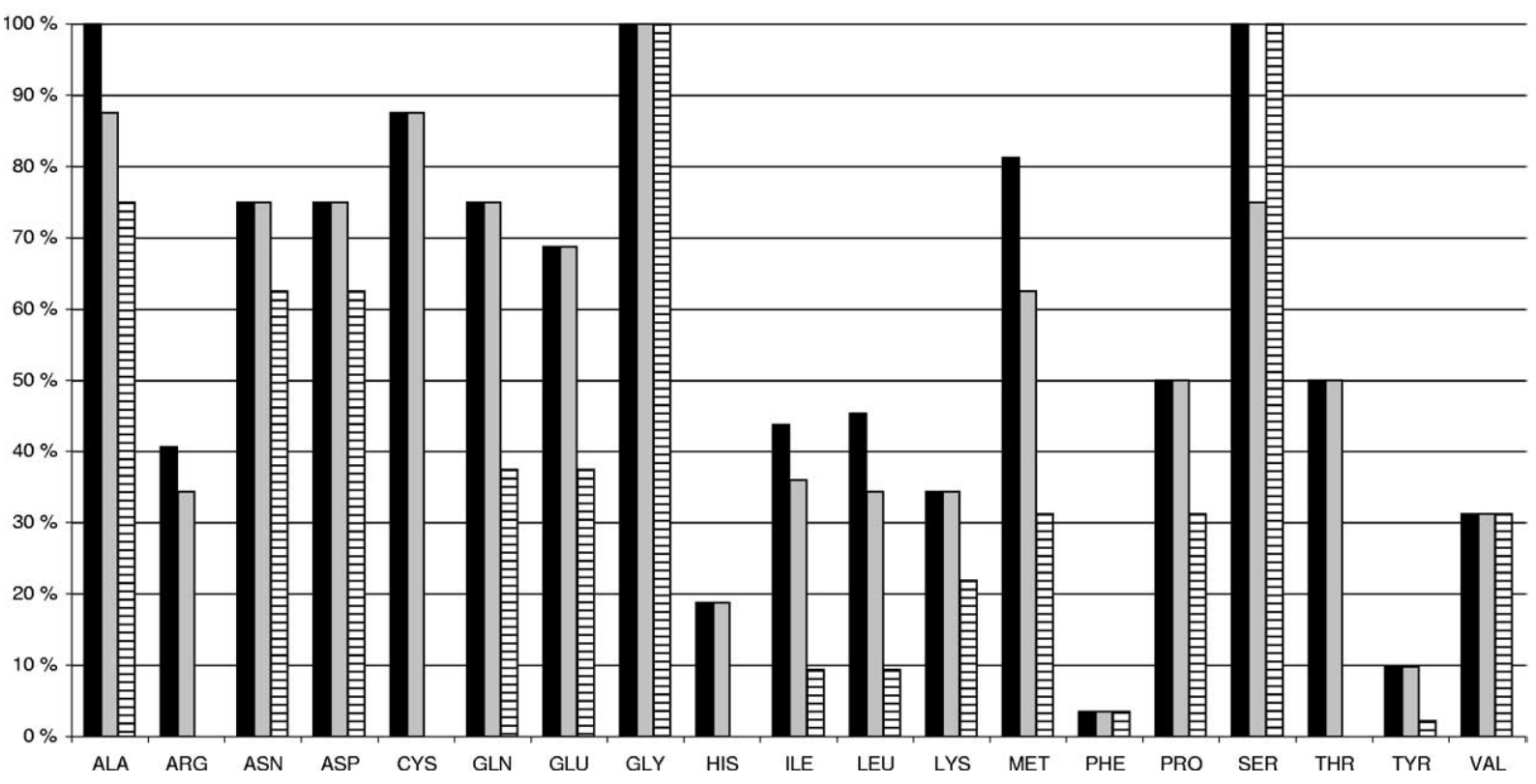

Fig. 5. Rank of the equation systems for amino acids obtained via the current method (leftmost column), the method by Rantanen et al. [15] (middle column) and the GC-MS method by Dauner and Sauer [2] (rightmost column). 
The new method can be seen to surpass both previous methods: It is never worse than the better than the competitors and is often better than either of them. The ability to handle overlaps helps the present method surpass the method by Rantanen et al. [15] in the analysis of six of the amino acids. In the case of three amino acids - alanine, glycine and serine - the new method is able to fully uncover the isotopomer distribution.

On the negative side, the large amino acids that contain ring structures or linear chains without sidegroups turn out to be difficult to all of the compared methods. This is due to the fact that such substructures tend to fragment poorly in the equipment used in these experiments.

\section{Discussion}

We have presented a method for the discovery of isotopomer distributions of molecules with tandem mass spectrometry. The method is able to deal with overlaps in the spectra, caused by fragments of near-equal mass, with the help of a precomputed database of relative intensities of different fragments.

When using the daughter ion scanning mode, the method was shown to be able to constrain the isotopomer distribution of different amino acids better than two existing methods. In particular, the isotopomer distributions of three amino acids, glycine, alanine and serine, can be fully uncovered with the method. It should be noted that the computational method is in no way tied to amino acids. In principle, tandem mass spectra of any small molecule can be analyzed.

In tests with isotope-labeled alanine it was found that multiple reaction monitoring mode using $15 \mathrm{eV}$ collision energy provided the best results. Daughter ion scanning mode with the same energy was slightly less accurate, and MRM with $30 \mathrm{eV}$ energy was clearly less accurate than the best method, indicating that the use of a correct energy level is critical in these kind of experiments.

For most of the amino acids - and for other molecules as well - the fragmentation in the tandem mass spectrometer is not sufficient to enable full discovery of isotopomer distribution: some carbons always appear together in the fragments, and no information is gained about the location of the labels. So-called $\mathrm{MS}^{n}$-mass spectrometers that are able to further fragment the molecule, do not suffer from this problem. However, such equipment is considerably more expensive than the equipment used here.

Another challenge to mass spectrometric methods is the analysis of intermediate metabolites. Their number alone makes it hard to develop such separation methods that the mass spectrum only consists of mass isotopomers of a single molecule. The method presented here needs to be extended to handle cases where the overlaps can occur between fragments of different molecules.

The described method for computing the positional isotopomer distribution of the molecule from the data produced by MS with daughter ion scanning is implemented as a MATLAB software called PIDC v2. The software takes as input the intensities of the full scan mode spectrum and daughter ion spectra and outputs the constraints to the positional isotopomer distribution. Both input and output are standard ASCII files. Database containing the relative amounts of different fragments for each molecule, MS device and energy level of interest is also in the form of ASCII file. The editing of the database can be done inside the PIDC software.

PIDC v2 is implemented and tested with version 6 of MATLAB in Windows and Linux operating systems, but probably works also with older versions of MATLAB and in different operating systems. The software will be made freely downloadable for academic use from the url http://www.cs.helsinki. fi/group/sysfys. 


\section{Acknowledgements}

PIDC v2 software was implemented as a software project exercise at the Department of Computer Science, University of Helsinki. We thank Taisto Gerdt, Timo Kääriäinen, Mika Miettinen, Pasi Rastas and Jari Tuominiemi for the skillful work and Katja Saarela for keeping the group on a good path. Financial support by Academy of Finland (SYSBIO programme) is gratefully acknowledged. The work of Juho Rousu was partially supported by the Individual Marie Curie Fellowship grant HPMF-CT-200202110.

\section{References}

[1] B. Christensen and J. Nielsen, Isotopomer analysis using GC-MS, Metabolic Engineering 1 (1999), E8-E16.

[2] M. Dauner and U. Sauer, GC-MS analysis of amino acids rapidly provides rich information for isotopomer balancing, Biotechnology Progress 16 (2000), 642-649.

[3] M. Dauner, J. Bailey and U. Sauer, Metabolic flux analysis with a comprehensive isotopomer model in Bacillus subtilis, Biotechnology and Bioengineering 76 (2001), 144-156.

[4] L. Di Donato, C. Des Rosiers, J.A. Montgomery, F. David, M. Garneau and H. Brunengraber, Rates of gluconeogenesis and citric acid cycle in perfused livers, assessed from the mass spectrometric assay of the ${ }^{13} \mathrm{C}$ labeling pattern of glutamate, Journal of Biological Chemistry 268 (1993), 4170-4180.

[5] New stable isotope-mass spectrometric techniques for measuring fluxes through intact metabolic pathways in mammalian systems: introduction of moving pictures into functional genomics and biochemical phenotyping, Metabolic Engineering 6(1) (2004), 85-100.

[6] M. Jeffrey, S. Roach, C. Storey, D. Sherry and C. Malloy, ${ }^{13} \mathrm{C}$ isotopomer analysis of glutamate by tandem mass spectrometry, Analytical Biochemistry 300 (2002), 102-205.

[7] M. Klapa, S.M. Park, A.J. Sinskey and G. Stephanopoulos, Metabolite and isotopomer balancing in the analysis of metabolic cycles: I. Theory, Biotechnology and Bioengineering 62 (1999), 375-391.

[8] W.-N. Lee, L.O. Byerley and E.A. Bergner, Mass isotopomer analysis: theoretical and practical considerations, Biological Mass Spectrometry 20 (1991), 451-458.

[9] H. Maaheimo, J. Fiaux, Z.P. Cakar, J.E. Bailey, U. Sauer and T. Szyperski, Central carbon metabolism of Saccharomyces cerevisiae explored by biosynthetic fractional ${ }^{13} \mathrm{C}$ labeling of common amino acids, European Journal of Biochemistry 268 (2001), 2464-2479.

[10] A. Marx, A. de Graaf, W. Wiechert, L. Eggeling and H. Sahm, Determination of the fluxes in the central metabolism of Corynebacterium glutamicum by nuclear magnetic resonance spectroscopy combined with metabolite balancing, Biotechnology and Bioengineering 49 (1996), 111-129.

[11] J.A. Morgan and D. Rhodes, Mathematical modeling of plant metabolic pathways, Metabolic Engineering 4(1) (2002), $80-89$.

[12] S.B. Noronha, H.J.C. Yeh, T.F. Spande and J. Shiloach, Investigation of the TCA cycle and the glyoxylate shunt in Escherichia coli BL21 and JM109 using ${ }^{13}$ C-NMR/MS, Biotechnology and Bioengineering 68 (2000), 316-327.

[13] G. Öz, P.G. Henry, E.R. Seaquist and R. Gruetter, Direct, noninvasive measurement of brain glycogen metabolism in humans, Neurochemistry International 43(4-5) (2000), 323-329.

[14] S. Park, M. Klapa, A.J. Sinskey and G. Stephanopoulos, Metabolite and isotopomer balancing in the analysis of metabolic cycles: II. Applications, Biotechnology and Bioengineering 62 (1999), 392-401.

[15] A. Rantanen, J. Rousu, R.A. Ketola, J.T. Kokkonen and V. Tarkiainen, Computing positional isotopomer distributions from tandem mass spectrometric data, Metabolic Engineering 4 (2002), 285-294.

[16] J. Rousu, A. Rantanen, H. Maaheimo, E. Pitkänen, K. Saarela and E. Ukkonen, A method for estimating metabolic fluxes from incomplete isotopomer information. Proc. Computational Methods in Systems Biology (CMSB), Lecture Notes in Computer Science 2602 (2003), 88-103.

[17] K. Schmidt, M. Carlsen, J. Nielsen and J. Villadsen, Modeling isotopomer distributions in biochemical networks using isotopomer mapping matrices, Biotechnology and Bioengineering 55 (1997), 831-840.

[18] K. Schmidt, L. Nørregaard, B. Pedersen, A. Meissner and J.Ø. Duus, Quantification of intracellular metabolic fluxes from fractional enrichment and ${ }^{13} \mathrm{C}-{ }^{13} \mathrm{C}$ coupling constraints on the isotopomer distribution in labeled biomass components, Metabolic Engineering 1 (1999), 166-179.

[19] G. Stephanopoulos, A. Aristidou and J. Nielsen, Metabolic Engineering: Principles and Methodologies, Academic Press, 1998. 
[20] T. Szyperski, Biosynthetically directed fractional ${ }^{13} \mathrm{C}$ labeling of proteinogenic amino acids. An efficient analytical tool to investigate intemediary metabolism, European Journal of Biochemistry 232 (1995), 433-448.

[21] T. Szyperski, R.W. Glaser, M. Hochuli, J. Fiaux, U. Sauer, J.E. Bailey and K. Würtrich, Bioreaction network topology and metabolic flux ratio analysis by biosynthetic fractional ${ }^{13} \mathrm{C}$ labeling and two-dimensional NMR spectroscopy, Metabolic Engineering 1 (1999), 189-197.

[22] W. Wiechert, M. Möllney, N. Isermann, M. Wurzel and A. de Graaf, Bidirectional steps in metabolic networks: III. Explicit solution and analysis of isotopomer labeling systems, Biotechnology and Bioengineering 66 (1999), 69-85.

[23] W. van Winden, C. Wittman, E. Heinzle and J. Heijnen, Correcting mass isotopomer distributions for naturally occuring isotopes, Biotechnology and Bioengineering 80 (2002), 477-479.

[24] C. Wittmann and E. Heinzle, Mass spectrometry for metabolic flux analysis, Biotechnology and Bioengineering 62 (1999), 739-750.

[25] C. Wittmann and E. Heinzle, Modeling and experimental design for metabolic flux analysis of lysine-producing corynebacteria by mass spectrometry, Metabolic Engineering 3 (2001), 173-191.

[26] C. Zupke and G. Stephanopoulos, Modeling of isotope distributions and intracellular fluxes in metabolic networks using atom mapping matrices, Biotechnology Progress 10 (1994), 489-498. 


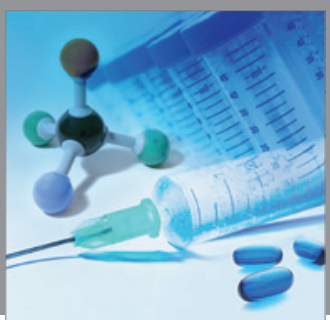

International Journal of

Medicinal Chemistry

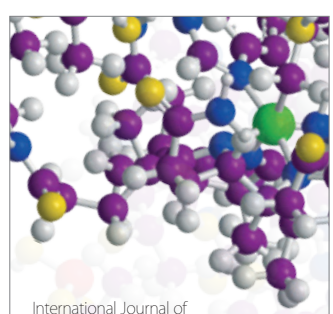

Carbohydrate Chemistry

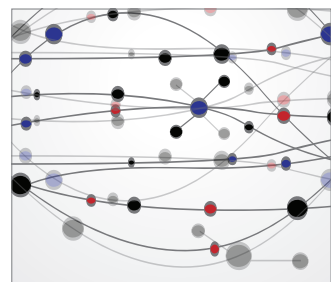

The Scientific World Journal
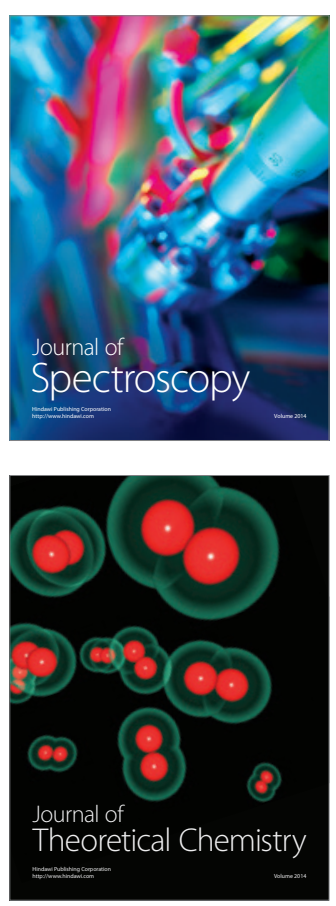
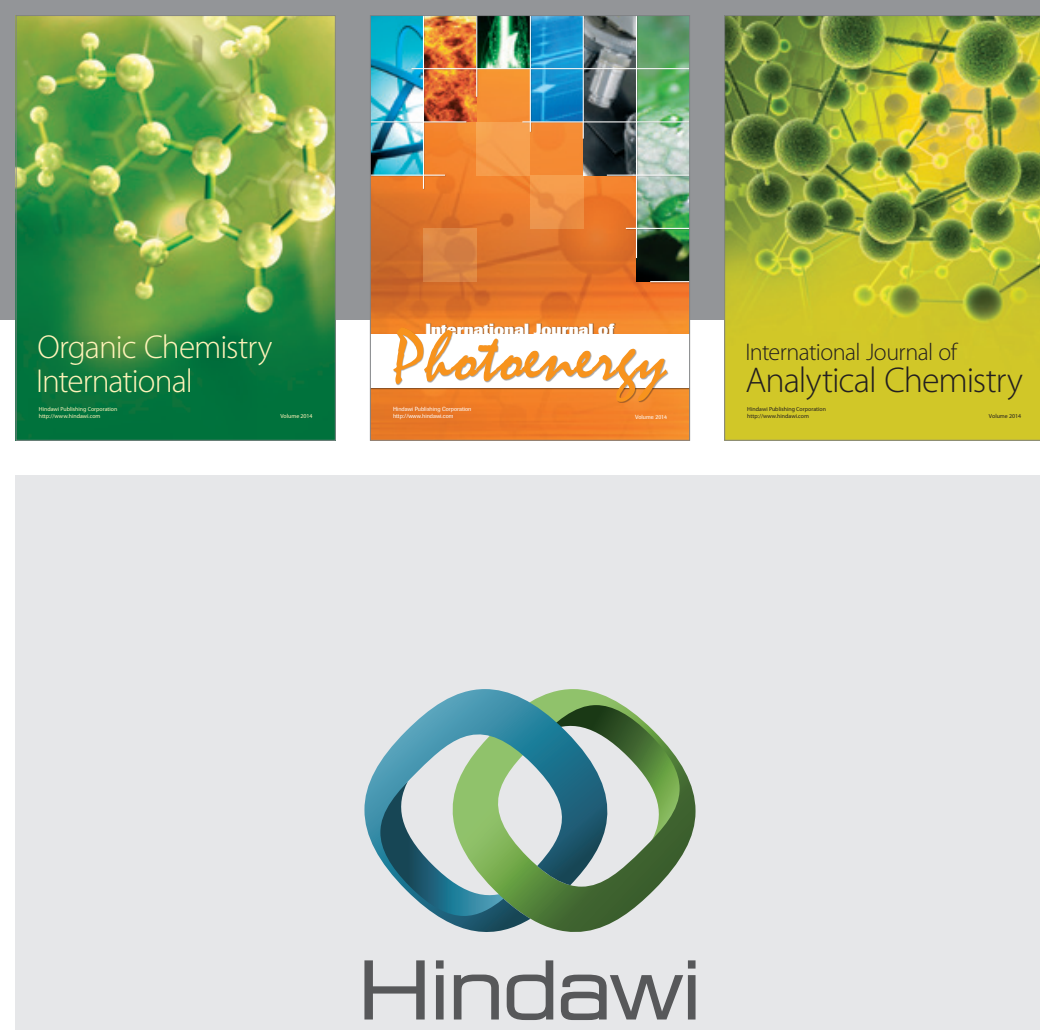

Submit your manuscripts at

http://www.hindawi.com
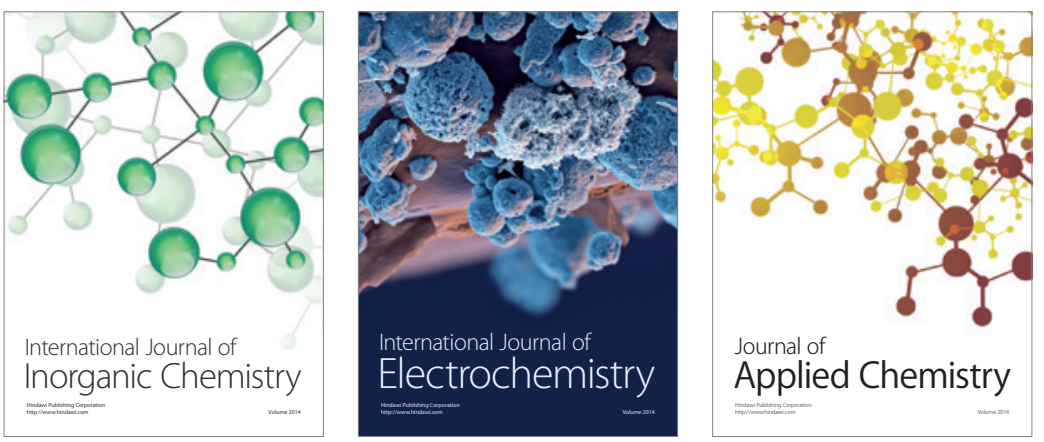

Journal of

Applied Chemistry
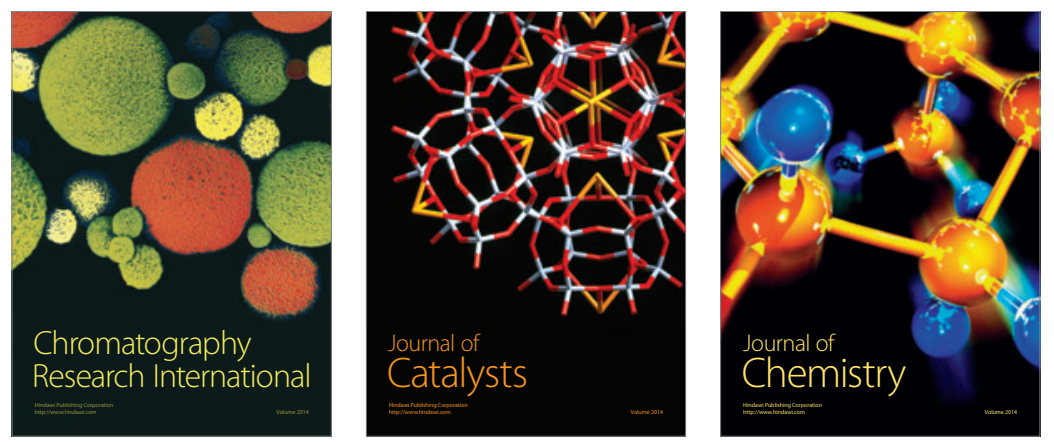
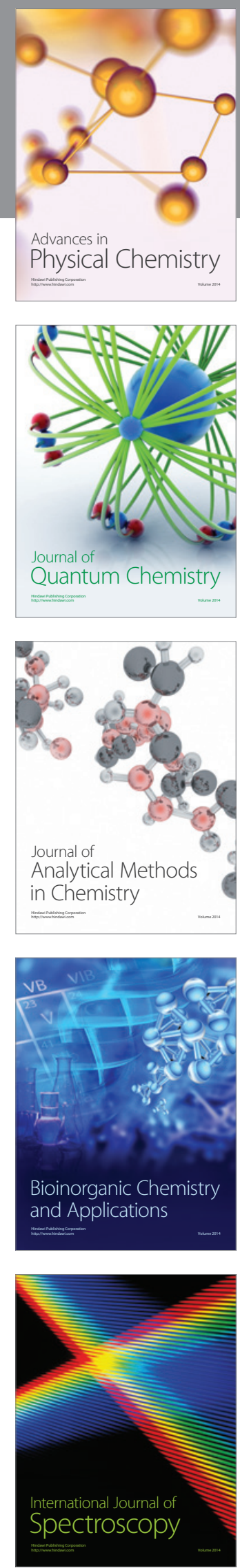